\title{
Effects of breast conservation on psychological morbidity associated with diagnosis and treatment of early breast cancer
}

\author{
LESLEY J FALLOWFIELD, MICHAEL BAUM, G P MAGUIRE
}

\begin{abstract}
Psychiatric morbidity was assessed in 101 women treated for early breast cancer $\left(T_{0,1,2}, N_{0,1}, M_{0}\right)$. Patients had expressed no strong preference for treatment, so were randomised to either mastectomy or breast conservation. The incidence of anxiety states or depressive illness, or both, among women whe underwent mastectomy was high (33\%) and comparable with that found in other studies. Slightly more of the patients who underwent a lumpectomy followed by radiotherapy had affective disorders, $38 \%$ having an anxiety state, depressive illness, or both.

These findings question the view that mutilating treatment is predominantly responsible for the measurable psychiatric morbidity reported previously. Counselling services should be provided for all women treated for breast cancer, not just those who undergo mastectomy.
\end{abstract}

\section{Introduction}

There is growing evidence that in early breast cancer relapse free intervals and survival rates are comparable in patients treated either by mastectomy or by local excision and radiotherapy. ${ }^{1}$ These data, together with reports that women who undergo mastectomy suffer high levels of anxiety or depression, or both, ${ }^{23}$ have led to an increasing trend towards more conservative surgery. In any case, many surgeons dislike performing the more mutilating operation. ${ }^{+}$

\footnotetext{
Cancer Research Campaign, Clinical Trials Centre, Rayne Institute, London SE5 9NU

LESLEY J FALLOWFIELD, BSC, DPHIL, research psychologist

Department of Surgery, King's College Hospital Medical School, London SE5 9NU

MICHAEL BAUM, CHM, FRCS, professor

Department of Psychiatry, University Hospital of South Manchester, West Didsbury, Manchester M20 8LR

G P MAGUIRE, FRCPSYCH, senior lecturer

Correspondence to: Dr Fallowfield.
}

While most clinicians believe intuitively that breast conservation will protect women against psychiatric morbidity, there has been little systematic study of the psychosocial outcome of this treatment. ${ }^{5.8}$ Two reports comparing psychological outcome of lumpectomy versus mastectomy for breast cancer found no appreciable differences between, or serious problems within, treatment groups, but they were studies conducted on small samples of patients. ${ }^{57}$ The authors of both papers also acknowledged that some emotionally distressed patients were not included. Another report described data from 38 patients randomised to treatment. ${ }^{6}$ Psychological problems were assessed using a non-standardised postal questionnaire. The only significant difference between treatment groups on 13 psychosocial variables was a less negative reaction to body image among the conservatively treated patients.

A recent study presented preliminary data on 40 patients who were helped to discuss treatment options and make their own choice. ${ }^{8}$ The authors concluded that giving the patient autonomy over her treatment or offering reconstruction when choice is not possible prevents psychiatric morbidity.

General conclusions about the relative psychological impact of different treatments are difficult to draw from these small studies, but they provide no clear evidence that lumpectomy rather than mastectomy necessarily lessens the likelihood of psychological problems. Possibly the advantages of conserving the breast are outweighed by greater worries about cancer ${ }^{9}$ and the adverse effects of radiotherapy. ${ }^{10-12}$ The Cancer Research Campaign's breast conservation trial $^{13}$ provided a unique opportunity to investigate these issues.

\section{Subjects and methods \\ SAMPLE}

Patients came from 10 of the 11 different centres participating in the Cancer Research Campaign's breast conservation trial. One centre chose to conduct its own psychological study. The protocol required participants to ascertain whether suitable patients - that is, women under 70 with early breast cancer-had a strong preference for mastectomy or breast conservation. If no preference was expressed the patient was randomly allocated to treatment by a telephone call to the clinical trials coordinator. The randomisation lists were generated by computer for individual participants, 
optimising the probability of a balanced treatment allocation for each participating clinician. All patients had a confirmed diagnosis of "early" stage I or II breast cancer- that is, tumours of $5 \mathrm{~cm}$ diameter or less with or without palpable axillary nodes and no evidence of distant metastasesand were judged by the surgeon to be eligible for either treatment strategy before randomisation

The proportion of suitable patients who were not entered into the trial will not be known until a full audit of the main study is done in every centre.

The original protocol envisaged a prospective parallel psychological arm to the main breast conservation trial, but the study of psychosocial sequelae began 24 months after the start of the main trial. As empirical work comparing psychological outcome of the different treatments was so sparse and no study of patients randomised to treatment had been published, we decided to initiate a retrospective study of all the patients already recruited.

There were 127 patients in the conservation trial potentially available for the psychological study; of these, seven died before interview, one was deemed physically too sick with recurrent disease to participate, nine refused or failed to reply to an invitation to join the study, and nine filled in questionnaires but declined to be interviewed. One hundred and one patients therefore took part in the retrospective study, of whom 48 were treated by local excision and radiotherapy and 53 underwent mastectomy; 34 of the patients given mastectomy also had radiotherapy. Four of the patients treated by lumpectomy and one treated by mastectomy had adjuvant chemotherapy.

\section{Method}

Psychiatric morbidity, sexual functioning, and social adjustment were determined postoperatively. A trained interviewer (LJF) first administered a semi-structured interview schedule designed to elicit information about the patient's marital state, key relationships, and use and availability of social support. The women were asked about their feelings on discovering the breast lump and their reaction to the diagnosis. They also recalled what doctors had told them about their diagnosis and treatment and whether the information was perceived as adequate. The interviewer then used the present state examination, ${ }^{14}$ minus the questions about psychotic symptoms, to determine the nature, frequency, and severity of symptoms of anxiety and depression in the four weeks before interview. DSM III diagnostic criteria ${ }^{15}$ were then applied to establish if there had been sufficient mood disturbance and other symptoms to justify a diagnosis of depressive illness or anxiety state. For example, to rate as a case of anxiety state a woman had to have had persistent feelings of tension and apprehension or inability to relax for at least four weeks plus four other symptoms, such as sleep disturbance, impaired concentration, fatigability, somatic symptoms (headaches, sweating, palpitations, tremor), panic attacks, or irritability. Likewise, to rate as depressed a woman had to have had persistent, unremitting unhappiness or lowering of mood for at least four weeks plus four other symptoms, such as sleep disturbance, impaired concentration, loss of self esteem, feelings of guilt, suicidal ideation, loss of energy, retardation or agitation, changes in appetite, weight loss or gain, or loss of libido.

Interviews were conducted in the patient's home, which permitted more intimate discussion than was possible on a busy ward and furthermore allowed the interviewer to assess the social environment, which might have contributed to problems experienced by the patient. All interviews lasted roughly 45 minutes (range 30-90 minutes) and were tape recorded. Ratererate reliability checks were performed on $30 \%$ of the tapes and showed ratings to have been consistent, with little drift. An independent rater checked the reliability of ratings for $10 \%$ of the interviews and found an overall agreement of $90 \%$.

Interviewing patients is time consuming and expensive and requires a trained interviewer. Another important part of this project was the validation of sound research tools that are cheap and easy to administer routinely to patients by paramedical personnel. Hence after the interview the women were given two self assessment questionnaires, the hospital anxiety and depression scale ${ }^{16}$ and the Rotterdam symptom check list. ${ }^{17}$ They completed these after the interviewer had left and returned them in a prepaid envelope to the trials centre. Compliance was $100 \%$ and a full analysis of the performance of these new questionnaires will be reported separately.

\section{COMPARABILITY OF GROUPS}

Table I shows the demographic characteristics of patients in the two treatment groups. Social class distributions and mean ages were similar. The times from operation to assessment of psychiatric morbidity varied in this retrospective study, but both the ranges and the mean times from operation to interview were similar in the two groups. Marital state differed between
TABLE I-Demographic characteristics

\begin{tabular}{|c|c|c|}
\hline & $\begin{array}{l}\text { Mastectomy group } \\
\quad(\mathrm{n}=53)\end{array}$ & $\begin{array}{l}\text { Lumpectomy group } \\
\qquad(n=48)\end{array}$ \\
\hline Mean age in years $(\mathrm{SD})$ [range] & $56(10)[32-72]$ & $58(8)[32-71]$ \\
\hline $\begin{array}{l}\text { Marital state: } \\
\text { Married } \\
\text { Single/widowed } \\
\text { Separated/divorced }\end{array}$ & $\begin{array}{r}35 \\
14 \\
4\end{array}$ & $\begin{array}{r}38 \\
6 \\
4\end{array}$ \\
\hline $\begin{array}{l}\text { Social class: } \\
\text { I } \\
\text { II } \\
\text { III (a) } \\
\text { III (b) } \\
\text { IV } \\
\text { V }\end{array}$ & $\begin{array}{r}8 \\
16 \\
11 \\
11 \\
7 \\
0\end{array}$ & $\begin{array}{r}5 \\
15 \\
13 \\
9 \\
6 \\
0\end{array}$ \\
\hline $\begin{array}{l}\text { Mean time from operation to interview in } \\
\text { months }(\mathrm{SD}) \text { [range] }\end{array}$ & $16 \cdot 7(7 \cdot 9)[4-32]$ & $15 \cdot 2(6 \cdot 6)[4-31]$ \\
\hline
\end{tabular}

the two groups, more single and widowed women having been treated by mastectomy. This random imbalance should therefore have favoured the $\partial$ breast conservation group slightly, as widowed and single people are more 3 . prone to depressive illness.

\section{Results and discussion}

\section{PSYCHIATRIC MORBIDITY}

Table II shows that according to our diagnostic criteria $11(21 \%)$ 을 of the patients with mastectomy had a depressive illness and $14 \mathrm{~N}$ (26\%) an anxiety state. Among the breast conservation patients $13 Z$ $(27 \%)$ were experiencing a depressive illness and $15(31 \%)$ an anxiety state. Some women $\left(8(15 \%)\right.$ in the mastectomy group, $10 \frac{\Phi}{3}$ $(21 \%)$ in the lumpectomy group) had both affective disorders. 8 These figures mean that overall an anxiety state or depressive illness, or both, was evident in $17(33 \%)$ of the patients with $\vec{\varnothing}$ mastectomy and $18(38 \%)$ of those treated by lumpectomy. Table $\%$ III shows the level of sexual interest experienced by the women in $\square$ the different treatment groups. On self assessment 18 of 48 of the patients with mastectomy (38\%) and 15 of 39 treated by lumpectomy (38\%) reported a lack of sexual interest. Five patients in the mastectomy group and nine in the lumpectomy group who $\mathbb{D}$ were elderly or single did not answer the question. We could not $\underset{\vec{O}}{\overrightarrow{2}}$ assess accurately the degree to which treatment might have been responsible for the decline in sexual interest as we had no preoperative measure of sexual activity.

\section{INFORMATION}

There are consistent reports that hospital patients feel inadequately informed about their diagnosis and treatment. ${ }^{18}$ One study

TABLE II-Psychiatric morbidity. Figures are numbers (percentages) of patients

\begin{tabular}{lcccc}
\hline & $\begin{array}{c}\text { Anxiety } \\
\text { state }\end{array}$ & $\begin{array}{c}\text { Depressive } \\
\text { illness }\end{array}$ & $\begin{array}{c}\text { Anxiety state } \\
\text { and depressive } \\
\text { illness }\end{array}$ & $\begin{array}{c}\text { Either or } \\
\text { both affective } \\
\text { disorders }\end{array}$ \\
\hline $\begin{array}{l}\text { Mastectomy group }(\mathrm{n}=53) \\
\text { Lumpectomy group }(\mathrm{n}=48)\end{array}$ & $14(26)$ & $11(21)$ & $8(15)$ & $17(32)$ \\
$13(31)$ & $13(27)$ & $10(21)$ & $18(38)$ \\
\hline
\end{tabular}

TABLE III-Self report on Rotterdam symptom check list assessing lack of sexual interest. Figures are numbers (percentages) of patients

\begin{tabular}{lcc}
\hline & "Not at all" to "a little" & "Somewhat" to "very much" \\
\hline Mastectomy group $(n=48)^{\star}$ & $30(62)$ & $18(38)$ \\
Lumpectomy group $(n=39)^{\star}$ & $24(62)$ & $15(38)$ \\
\hline
\end{tabular}

*Fourteen elderly or single women in the two groups without sexual partners did not answer question. 
of patients with mastectomy found that $21 \%$ were dissatisfied with the information given. ${ }^{1}$ Most of the ethical committees of the centres participating in our study required surgeons to obtain informed consent from patients before randomisation. It would therefore be reasonable to hope that patients in this trial would have had more information about their illness and surgical options than is perhaps always possible in a busy outpatient department. Table IV shows that in answer to the question "Do you feel that the information that you received at the hospital was adequate?" over half of the

TABLE IV-Perception of information received. Figures are numbers (percentages) of patients

\begin{tabular}{llll}
\hline & \multicolumn{2}{c}{$\begin{array}{c}\text { "Do you feel that the information that you received } \\
\text { at the hospital was adequate?" }\end{array}$} & Total \\
\cline { 2 - 3 } & Yes & No & $52(100)^{\star}$ \\
\hline Mastectomy group & $26(50)$ & $26(50)$ & $48(100)$ \\
Lumpectomy group & $22(46)$ & $26(54)$ & 100 \\
\hline Total & 48 & 52 & \\
\hline
\end{tabular}

* One patient did not answer question.

women $(52 \%)$ said "no." There was no significant difference in this perception of information received between patients in the mastectomy and lumpectomy groups. We had hypothesised that the incidence of anxiety states and depressive illness would be less for patients who perceived information to have been good. This appeared to be so, cases of anxiety or depression, or both, being recorded in 11 of 48 women $(23 \%)$ who reported the level of information to have been good as compared with 24 out of 52 women $(46 \%)$ who were poorly informed. This difference was significant $\left(\chi^{2}\right.$ test; $\mathrm{p}<0.03)$. Unfortunately, we cannot tell whether these data mean that anxious patients are less satisfied with their information or well informed patients are less anxious. This direction of causality is an important issue to address to improve communication between doctors and patients with breast cancer and demands further investigation in a prospective study.

While the proportions of women suffering from an affective disorder were similar in the two groups, their problems seemed to be qualitatively different.

\section{ANXIETY}

The anxiety exhibited by women treated by lumpectomy seemed to stem from a feeling of uncertainty about the prognosis and the constant awareness of possible recurrence or metastatic disease. Some of these patients admitted to compulsive checking of their breasts for other lumps; five said that they examined themselves more than once a day. The following extracts are from the taped interviews of two patients:

"Do you know, if I get a pain in my stomach now it's got to be cancer; if I've got a pain in my back I'm going to get lung cancer; get a bad head, I'm getting a tumour on the brain. I just can't get it out of my head."

"I just cannot keep my hand off [my breast]. It's something that I've never ever done. I spend hours just lying there feeling, and when I touch I feel as if I've got lumps all over."

Anxious patients with mastectomy obviously shared those uncertainties but expressed more overt concern about the effect of their surgery on relationships and a heightened self consciousness about appearance. None of the patients with mastectomy admitted to daily self examination, though three said that they checked their remaining breast at least weekly. An item in the Rotterdam symptom check list questionnaire asked how dissatisfied patients felt about the appearance of their scars; seven of the patients with mastectomy were very or somewhat dissatisfied compared with one of the patients treated by lumpectomy $(p=0.04)$. Many of the women who had had breast conservation seemed less certain than the patients in the mastectomy group that they had received the right operation, though this is difficult to quantify directly. Possibly the surgeons themselves still had a subthreshold doubt, which unwittingly they were communicating to the patient.

\section{DEPRESSION}

There were also differences between the groups in the reasons given for becoming depressed. Patients treated by lumpectomy complained about the particularly unpleasant and denervating fatigue that persisted after radiotherapy. Only two reported suffering no side effects. They felt dispirited at their slow return to health and normal activity after such a seemingly small operation. Conversely, many of the patients with mastectomy had had less expectation of a rapid return to work and normal functioning, and some therefore reported surprise that the treatment had not been quite as bad as they had feared. Of the 34 patients in the mastectomy group who had to have radiotherapy, seven were depressed. One patient in our study had great difficulty in adjusting to her mastectomy. Her intractable depression necessitated admission to a psychiatric hospital for electroconvulsive therapy.

\section{SEXUAL PROBLEMS}

While rejection by a spouse or partner is not uncommon after mastectomy, this happened to only one patient in our sample. We have no preoperative measures of the level of sexual activity of patients in this retrospective study, but over one third of the women in both groups reported a loss of sexual interest since their diagnosis and treatment for breast cancer. Loss of libido is a common symptom of depressive illness, ${ }^{15}$ but many people have assumed that the severe assault on body image and self esteem produced by amputation of the breast is in itself sufficient cause for the deterioration of sexual activity. ${ }^{19}$ As the patients treated by lumpectomy in our study had just as severe a decline in their desire for love making as the patients in the mastectomy group, possibly such assumptions need rethinking. Of the depressed patients with mastectomy three of 11 reported loss of libido compared with seven of the 13 depressed patients treated by lumpectomy. Such numbers were not significantly different, but there was a suggestion that loss of libido was symptomatic of depression in the lumpectomy treated patients but more linked with inhibitions, loss of femininity, self esteem, and body image concomitant with breast loss in the more radically treated group.

\section{CHOICE OF TREATMENT}

Possibly the women in this study who were prepared to be randomised to treatment displayed rather different psychological characteristics from patients who prefer to participate in the decision making process. Women with a greater sense of autonomy might well adapt more readily to the consequences of a treatment policy that they have chosen. Evidence from a very small sample of women suggests that careful preoperative assessment and help with decision making reduce the levels of psychiatric morbidity that we have reported. ${ }^{8}$

\section{Conclusions}

This retrospective study suggests that the suppositions that $(a)$ all women with breast cancer wish to retain their breast and $(b)$ that breast conservation prevents psychiatric morbidity might well be misplaced. It was not unusual at interview for patients in the lumpectomy group to express deep concern that they had received the "wrong" operation and to comment that they felt constantly worried that the surgeon might not have removed all the cancer and that it would return. If so far as survival and local control of disease are concerned it really is entirely equivocal which treatment is 
offered in early breast cancer then it becomes of paramount importance to consider the psychological impact of treatment to be offered.

The degree of psychosocial morbidity among the patients treated by local excision and radiotherapy was a disappointing finding, but one which cannot be ignored. These women clearly need just as much counselling support as patients who undergo mastectomy.

As more surgeons start to advocate breast conservation it is important that we have more basic research into the causal factors of the psychiatric morbidity experienced by women who receive this treatment.

We thank all the surgeons and radiotherapists who permitted access to their patients and the Cancer Research Campaign for financial support.

\section{References}

1 Fisher B, Bauer $M$, Margolese R. Five year results of a randomised clinical trial comparing total mastectomy and segmental mastectomy with or without radiation in the treatment of breas cancer. N Engl f Med 1985;312:665-73.

2 Morris T, Steven-Greer H, White P. Psychological and social adjustment to mastectomy (a 2-year follow-up study). Cancer 1977:40:2381-7.

3 Maguire GP, Lee EG, Bevington DJ, et al. Psychiatric problems in the year after mastectomy. $B r$ Med f 1978;i:963-5.

4 Ray C, Baum M. Psychological aspects of early breast cancer. New York: Springer-Verlag Inc, 1985

5 Sanger CK, Reznikoff $M$. A comparison of the psychological effects of breast-saving procedures with the modified mastectomy. Cancer 1981;48:2341-6.
6 Schain W, Edwards BK, Gorrell CR, et al. Psychosocial and physical outcomes of stage I breast cancer therapy: mastectomy $\mathrm{v}$ excisional biopsy and irradiation. Breast Cancer Res Treat

7 Steinberg MD, Juliano MA, Wise L. Psychological outcome of lumpectomy versus mastectomy in the treatment of breast cancer. Am $\mathcal{F}$ Psychiatry 1985;142:34-9.

8 Ashcroft JJ, Leinster SJ, Slade PD. Breast cancer-patient choice of treatment: preliminary $\widehat{\Omega}$ communication. I R Soc Med 1985;78:43-6.

9 Senescu RA. The development of emotional complications in the patient with cancer. $f$ Chronic Dis 1963;16:813-32.

10 Peck A, Boland J. Emotional reactions to radiation treatment. Cancer 1977;40:180-4.

11 Forester BM, Kornfeld RS, Fleiss J. Psychiatric aspects of radiotherapy. Am 7 Psychiatry 1978;135:960-3.

12 Holland JC, Rowland J, Lebovits A, Rusalem R. Reactions to cancer treatment: assessment of .emotional response to adjuvant radiotherapy as a guide to planned intervention. Psychiatr Clin $\overrightarrow{\overrightarrow{\vec{B}}}$ North Am 1979;2:347-58.

13 Peckham MJ (chairman). Protocol for a collaborative trial to evaluate the need for mastectomy in the management of early breast cancer. London: Cancer Research Campaign Clinical Trials Centre, manase

14 Wing JK, Cooper JE, Sartorious N. Measurement and classification of psychiatric symptoms. Cambridge: Cambridge University Press, 1974.

15 American Psychiatric Association. Diagnostic and statistical manual of mental disorder. 3rd ed. ®๐ Washington, DC: APA, 1980.

16 Zigmund AS, Snaith RP. The hospital anxiety and depression scale. Acta Psychiatr Scand ڤั 1983;67:370.

17 Pruyn JFA, Maguire P, De Haes JCJM. Two methods of measuring some aspects of quality of life. In: Quality of life methods of measurement and related areas. Proceedings of the second EORTC quality of life workshop. Copenhagen: European Organisation for the Research and Treatment of Cancer, 1981:52-5.

18 Ley P. Giving information to patients. In: Eiser JR, ed. Social psychology and behavioral science. Chichester: John Wiley and Sons Ltd, 1982:339-71.

19 Bransfield DD. Breast cancer and sexual functioning: a review of the literature and implications for future research. Int I Psychiatry Med 1982-3;12(3):197-211.

(Accepted 16 September 1986)

\title{
Optimising antiemesis in cancer chemotherapy: efficacy of continuous versus intermittent infusion of high dose metoclopramide in emesis induced by cisplatin
}

\author{
PAMELA S WARRINGTON, SIMON G ALLAN, MICHAEL A CORNBLEET, \\ JANET S MACPHERSON, JOHN F SMYTH, ROBERT C F LEONARD
}

\begin{abstract}
Thirty three untreated patients being given cisplatin received metoclopramide $(7 \mathrm{mg} / \mathrm{kg}$ ) for antiemesis by either continuous or intermittent infusion in a random order. Each patient received intravenous dexamethasone in addition. High pressure liquid chromatography was used to measure plasma concentrations of metoclopramide. The two regimens were evaluated for antiemetic efficacy and the incidence of side effects.

The intermittent metoclopramide regimen resulted in peak and trough plasma concentrations of metoclopramide with accumulation at eight hours, while the loading dose and continuous infusion resulted in mean plasma concentrations greater than $0.85 \mu \mathrm{g} / \mathrm{ml}(2.8 \mu \mathrm{mol} / \mathrm{l})$ throughout the eight hour period. The continuous infusion was associated with a significant improvement in nausea and vomiting and reduction in diarrhoea.
\end{abstract}

\footnotetext{
Imperial Cancer Research Fund Medical Oncology Unit, University Department of Clinical Oncology, Western General Hospital, Edinburgh EH4 2XU PAMELA S WARRINGTON, MSC, MPS, staff pharmacist SIMON G ALLAN, MB, MRCP, research fellow

MICHAEL A CORNBLEET, MD, MRCP, senior registrar

JANET S MACPHERSON, BSC, research officer

JOHN F SMYTH, MD, FRCP, professor of medical oncology

ROBERT C F LEONARD, MD, FRCPED, senior lecturer
}

Correspondence and requests for reprints to: Mrs Warrington.
Major control of emesis (two episodes or fewer) was achieved in 27 patients receiving continuous metoclopramide compared with 18 receiving intermittent metoclopramide.

\section{Introduction}

Current chemotherapy strategies still of necessity use highly emetogenic drugs. Clinical experience together with psychological studies confirm that the gastrointestinal sequelae of chemotherapy are of paramount importance. ${ }^{\prime}$ Cisplatin is one of the most emetogenic compounds known but is widely used in curative and $N$ palliative chemotherapy for cancer. Only recently have effective $\rightarrow$ antiemetics been developed, and much effort has been focused on the control of emesis induced by cisplatin: high dose meto- $N$ clopramide given by intermittent infusion has proved useful, ${ }^{2-5}$ and N we showed that dexamethasone significantly enhances its antiemetic effect. ${ }^{6}$ In our previous study, however, only $65 \%$ of patients achieved major control (two or fewer episodes of vomiting); further improvement is required. Using an intermittent regimen, Meyer $\stackrel{\mathcal{\Phi}}{\rightarrow}$ et al found that effective antiemesis required a minimum plasma metoclopramide concentration of $0.85 \mu \mathrm{g} / \mathrm{ml}(2 \cdot 8 \mu \mathrm{mol} / \mathrm{l})$.

High dose intermittent infusion as reported by Gralla et al results not only in wide variations in plasma concentrations of the drug but also in accumulation. ${ }^{38}$ As a relation between plasma concentrations and antiemetic effect has been described failure of control may be the result of subtherapeutic concentration of metoclopramide at a critical time in the onset of vomiting, which is usually within two hours after the administration of cisplatin. 